\title{
Uso racional de antibióticos y tecnología FilmArray para identificación rápida de bacteriemias en unidad de cuidados intensivos pediátrica
}

\section{Rational use of antibiotics and FilmArray technology for rapid identification of bacteremias in a pediatric intensive care unit}

\author{
Liliana Mazzillo Vega ${ }^{a}$, Nancy Cabrera Bravo ${ }^{a}$
}

${ }^{a}$ Hospital Infantil Los Ángeles. Nariño, Colombia.

Recibido: 27 de septiembre de 2019; aceptado: 17 de mayo de 2020

¿Qué se sabe del tema que trata este estudio?

Existen gran cantidad de estudios realizados en población adulta sobre la utilidad de programas de uso racional de antibióticos y pruebas rápidas para la identificación de bacteriemias en pacientes críticos, sin embargo, hay pocos estudios en la población pediátrica.
¿Qué aporta este estudio a lo ya conocido?

El presente estudio afirma la utilidad de identificar tempranamente los microorganismos causantes de bacteriemias que junto al programa de uso racional de antibióticos permiten al médico tratante formular terapias antibióticas dirigidas disminuyendo la exposición de antibióticos empíricos en niños críticos.

\section{Resumen}

Las infecciones graves son la principal causa de ingreso a cuidados intensivos pediátricos. El panel FilmArray BCID permite identificar rápidamente a microorganismos causantes de bacteriemias. $\mathbf{O b}$ jetivo: evaluar la eficacia de la identificación rápida de microorganismos asociado a un Programa de Uso Racional de Antibióticos (URA) en reducir los tiempos de terapias antibióticas, en un hospital pediátrico. Pacientes y Método: Estudio retrospectivo, que incluyó 100 pacientes, en su primer episodio de bacteriemia, divididos en 2 grupos de 50 cada uno: Intervención (FilmArray BCID y programa URA) y Controles históricos pareados para la misma especie del microrganismo identificado (microbiología convencional). Las variables evaluadas fueron los tiempos de identificación microbiana, latencia de la terapia dirigida y de desescalar antibióticos. Resultados: Los grupos fueron comparables en características demográficas, foco de infección y etiología de bacteriemia. El tiempo promedio de
Palabras clave:

Bacteriemia;

Cuidados Intensivos;

Pediatría;

Antibióticos;

Diagnóstico molecular

Correspondencia:

Liliana Mazzillo Vega

lilianamazzillo@gmail.com 
identificación de microorganismos fue de 23 h (IC 95\% 12,4-26,7) en el grupo intervención, y 70,5 h (IC 95\% 65,2-78,6) en el control ( $\mathrm{p}<0,05)$, mientras que la latencia de inicio de terapia dirigida fue de 27,9 h (IC 95\% 22,3-32,8) y 71,9 h (IC 95\% 63,2-77,8) respectivamente ( $<<0,05)$. El tiempo de desescalar o suspender antibióticos fue de 6,4 h (IC 95\% 2,76-9,49) y 22 h (IC 95\% 6,74-35,6) en los grupos mencionados $(\mathrm{p}>0,05)$. Conclusión: El panel FilmArray BCID articulado a un programa URA, contribuye a la identificación de los microorganismos causantes de bacteriemias en menor tiempo que los métodos convencionales, siendo una herramienta que optimiza las terapias antibióticas en niños críticamente enfermos.

\section{Abstract}

Severe infections are the leading cause of admission to pediatric intensive care. The FilmArray BCID panel quickly identifies microorganisms that cause bacteremia. Objective: To evaluate if the rapid identification of the microorganisms that cause bacteremia, along with a Rational Use of Antibiotics (RUA) Program, allows optimizing the time of antibiotic therapy in a pediatric hospital. Patients and Method: Retrospective study which included 100 patients presenting their first episode of bacteremia, divided into 2 groups of 50 each. The first one was Intervention (FilmArray BCID and RUA program) and the second one was Historical Controls (conventional automated ID/AST). The variables evaluated were the time required for microbial identification, duration of appropriate therapy, and antibiotic de-escalation. Results: The groups were comparable in terms of demographic characteristics, focus of infection, and etiology of bacteremia. The average time of microorganisms' identification of the control group was 70.5 hours (IC 95\% 65.2-78.6) and 23.0 hours (IC 95\% 12.4$26.7)$ in the intervention one $(\mathrm{p}<0.05)$. The average time of targeted therapy onset was shorter in the intervention group (27.9 $\mathrm{h}$ [IC 95\% 22.3-32.8]) than that of the control one $(71.9 \mathrm{~h}$ [IC 95\% 63.2-77.8]) $(\mathrm{p}<0.05)$. Finally, the time to de-escalate or discontinue antibiotics in the intervention group and the control one was 6.4 hours (IC 95\% 2.76-9.49) hours and 22.0 hours (IC 95\% 6.74-35.6 h) respectively $(\mathrm{p}>0.05)$. Conclusion: The FilmArray panel along with the RUA Program allows the identification of the microorganisms causing bacteremia faster than conventional methods, which positions it as a tool that optimizes antibiotic therapy of critical patients.
Keywords:

Bacteremia;

Pediatric Critical Care;

Antibiotics;

Molecular Diagnosis

\section{Introducción}

La identificación temprana de microorganismos que ocasionan bacteriemias en pacientes críticos pediátricos podría permitir optimizar las terapias antimicrobianas y favorecer los resultados de morbimortalidad $^{1}$, disminuyendo la exposición innecesaria a múltiples antibióticos empíricos que, en su gran mayoría, son de amplio espectro y pueden contribuir a incrementar, tanto la resistencia bacteriana como el riesgo de presentar eventos adversos asociados a medicamentos ${ }^{2,3}$.

Anteriormente, no se disponía de herramientas tecnológicas que permitieran identificar rápidamente los patógenos causantes de las infecciones del torrente sanguíneo, y se iniciaban antibióticos empíricos de amplio espectro por 48 a $72 \mathrm{~h}$, hasta identificar al responsable, y dirigir la terapia antibiótica (continuar, escalar, desescalar o suspender). A nivel mundial existe una escasa implementación de programas de uso racional de antibióticos (URA) en población pediátrica exponiendo al paciente a los riesgos mencionados, $y$ a la inoportunidad en la toma de decisiones. Esta si- tuación ha mejorado en la actualidad debido a que los hospitales han implementado progresivamente programas URA ${ }^{2}$.

Actualmente existen varias tecnologías que disminuyen el tiempo de identificación de los patógenos causantes de las bacteriemias, entre ellas el panel de identificación de hemocultivos por PCR FilmArray BCID. Sin embargo, son escasos los estudios pediátricos que evalúan su efectividad en cuanto a la oportunidad y precisión de la terapia antibiótica, así como su impacto en los resultados clínicos en el manejo de las bacteriemias. Los estudios disponibles, predominantemente en adultos, resaltan la importancia de articular las pruebas de identificación rápida al uso racional de antibióticos, la comunicación efectiva entre el laboratorio clínico con los médicos tratantes y el mejorar la oportunidad en la interpretación de los resultados del panel, actividades que contribuyen a lograr resultados óptimos del programa, e implicando un personal de salud previamente sensibilizado y capacitado para evitar las barreras en su implementación. Dicho programa debe obtener y analizar indicadores de calidad para medir el impacto de su im- 
plementación en las instituciones hospitalarias ${ }^{2,4-6}$. El panel FilmArray BCID se implementó en el Hospital Infantil Los Ángeles (HILA), en mayo de 2017. Esta tecnología permite identificar 24 patógenos al nivel de la especie o del género (bacterias Gram positivas, bacterias Gram negativas, hongos) y 4 genes de resistencia antibiótica (van A/B, blaKPC y mecA) en aproximadamente 1 hora después de que el hemocultivo se ha positivizado. Nuestro objetivo fue evaluar si la identificación rápida de los microorganismos en bacteriemias articulada al programa URA, permite optimizar el tiempo de terapia antibiótica dirigida y el tiempo de desescalar antibióticos en una Unidad de Cuidado Intensivo Pediátrica (UCIP) de una institución de alta complejidad.

\section{Pacientes y Método}

Estudio cuantitativo experimental realizado en el Hospital Infantil Los Ángeles en Pasto, departamento de Nariño, Colombia. El Programa URA fue implementado desde 2016. El proyecto fue avalado por el Comité de Ética del local el 9 de octubre de 2018, siendo clasificado como "Bajo Riesgo" de acuerdo con el artículo 11 de la Resolución 8430 de 1993.

Se incluyeron 100 pacientes con primer episodio de bacteriemia, con edad entre 1 mes y 18 años, requiriendo ser hospitalizados en UCIP.

El grupo Intervención consideró 50 pacientes en quienes se realizó todos los paneles FilmArray BCID entre mayo de 2017 y enero de 2019, mientras que el grupo control 50 pacientes con microbiología convencional; estos fueron controles históricos reclutados entre enero de 2014 y diciembre de 2016, pareados para la misma especie del microrganismo identificado.

Se excluyeron los pacientes que fallecieron, o que fueron remitidos antes que el hemocultivo fuera reportado, y aquellos con historia clínica incompleta. Los sesgos de información se controlaron realizando una búsqueda exhaustiva de historias clínicas. Se estandarizaron las definiciones de las variables, se creó un proceso organizado para la revisión de las historias clínicas, con codificaciones en la recogida de datos, realizándose una prueba piloto.

En ambos grupos se realizaron los hemocultivos utilizando Bactec Plus Aeróbico (BD Diagnostics, Franklin Lakes, Estados Unidos) y se efectuó la coloración de Gram a partir de las muestras de botellas con crecimiento positivo, y un subcultivo en agar, para luego identificar los microorganismos por método automatizado Phoenix100 (BD Diagnostics, Franklin Lakes, Estados Unidos). En el grupo intervención se realizó el panel FilmArray BCID (Biofire, Salt Like City, Estados Unidos) según las indicaciones del fabri- cante luego de la identificación del microorganismo por la tinción de Gram.

Los datos, se recolectaron de una fuente secundaria (historias clínicas sistematizadas). Se consignaron variables clínicas y sociodemográficas, sensibilidad y especificidad de la prueba FilmArray. Se identificaron los microorganismos por microbiología convencional y FilmArray. La variable de exposición fue la técnica de la microbiología convencional y la tecnología FilmArray articulada al programa URA. La variable de respuesta fue el tiempo de exposición. Se incluyeron otras covariables para hacer ajuste de los análisis, como Tiempo de identificación del microorganismo por hemocultivo o por FilmArray (tiempo promedio en horas desde la toma del hemocultivo hasta el reporte por microbiología del hemocultivo o del FilmArray), tiempo de terapia dirigida (tiempo promedio en horas desde la toma del hemocultivo hasta que se inició el antibiótico apropiado en ambos grupos) y tiempo de desescalar (tiempo promedio en horas desde la identificación del microrganismo por hemocultivo o por FilmArray hasta la suspensión o disminución en espectro de un antibiótico empírico que no era apropiado para el germen causante de la bacteriemia). Se definió terapia empírica inapropiada si los antibióticos se iniciaron desconociendo el germen causante, pero que luego de conocer el resultado del FilmArray o del hemocultivo, el médico decide que no se requería o era inapropiado.

Análisis de la información: Estadística descriptiva expresada en frecuencias relativas y absolutas y gráficas para variables cualitativas, y media y desviación estándar para variables cuantitativas. La normalidad se testeó con la prueba de Kolmogorov-Smirnov. Se utilizó prueba estadística chi-cuadrado y prueba T Student, para las variables cualitativas y cuantitativas de distribución paramétrica, y prueba U de Mann-Whitney cuando la distribución fue no paramétrica. Las pruebas fueron realizadas con un $95 \%$ de intervalo de confianza. Se utilizó el software SPSS ${ }^{\circledR}$, version 21 (IBM Corp. Released 2012. IBM SPSS Statistics for Windows, Version 21.0. Armonk, NY: IBM Corp.).

\section{Resultados}

Se reclutaron 50 pacientes en cada grupo, siendo ellos comparables en características demográficas, foco de infección y etiología de bacteriemia. En ambos grupos, las bacteriemias fueron predominantemente asociadas a una infección pulmonar (tabla 1).

Respecto a su microbiología, los cultivos para gram positivos corresponden al 50\%, gram negativos $49,5 \%$ y hongos el 0,5\%. Los cultivos fueron polimicrobianos en el $11 \%(11 / 100)$, tendiendo a ser mayor en el grupo intervención $(14 \%, 7 / 50)$. No existieron diferencias 
Tabla 1. Características sociodemográficas y clínicas de niños con bacteriemia

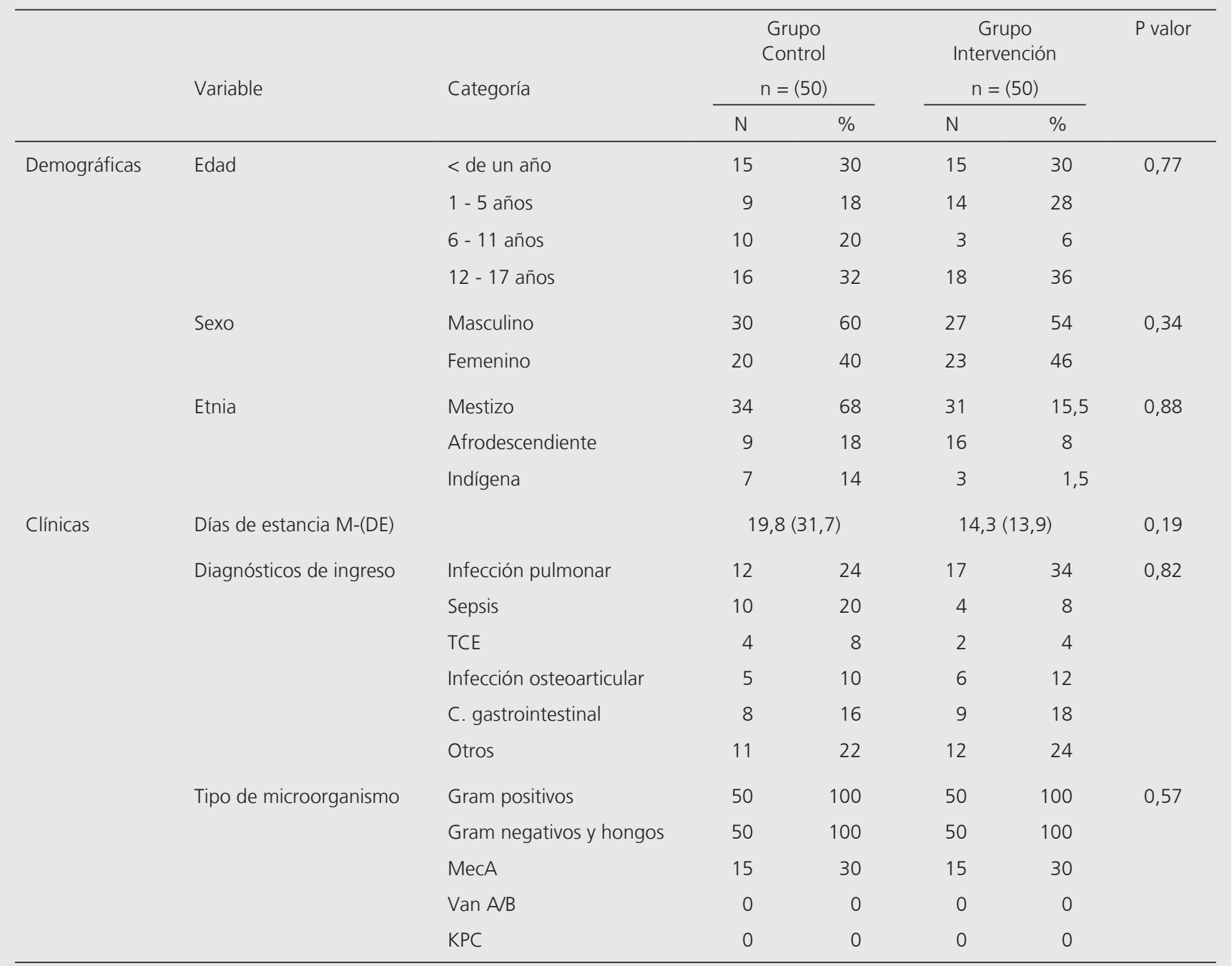

TCE: Trauma craneoencefálico; C. gastrointestinal: Cirugía gastrointestinal; MecA: Gen responsable de la resistencia a la meticilina; Van A/B: Determinantes genéticos de resistencia a glucopéptidos; KPC: Carbapenemasa clase A, confiere resistencia a todos los betalactámicos. *Valor de Chi 2 (valor de $p<0,05$ ). ${ }^{*}$ Prueba U de Mann Whitney para variables cuantitativas no normales $(p<0,05)$.

significativas en la distribución de microorganismos entre los grupos, siendo las bacterias más frecuentes Staphylococcus aureus, Staphylococcus coagulasa negativo, Klebsiella pneumoniae, Escherichia coli y Serratia marcescens (tabla 2).

El tiempo promedio de identificación del microorganismo fue menor en el grupo intervención respecto al control $[23,1 \mathrm{~h}$ (IC 95\% 12,4-26,7) vs 70,5 h $(65,2-$ $78,6) \mathrm{p}<0,05]$, al igual que el tiempo promedio de inicio de terapia dirigida $[27,9 \mathrm{~h}$ (IC $95 \% 22,3 \mathrm{~h}-32,8) v s$ 71,9 h (IC 95\% 63,2-77,8 h) p < 0,05]. Hubo una tendencia a un menor tiempo de desescalar o suspender antibióticos en el grupo intervención [6,4 h (IC 95\% 2,76-9,49 vs 6,74-35,6, p > 0,05], siendo más acentua- do en las bacterias gram positivas [2,1 (IC 95\% 0,113,7 ) vs $13,1 \mathrm{~h}$ (IC 95\% 3,2-27,4 h, p > 0,05) que en gram negativas 11,2 (IC 95\% 4,0-16,3) vs 32,9 (IC 95\% $5,12 \mathrm{~h}-55,4 \mathrm{~h} \mathrm{p}>0,05)$ (tabla 3 y figura 1). La desescalación o suspensión de antibióticos luego de interpretar el FilmArray ocurrió en un $100 \%$ de los pacientes, a diferencia del grupo control, donde solo se realizó en el $76 \%$.

La tecnología FilmArray tuvo sensibilidad y especificidad de 97,4\%, en mono y policultivos (tabla 4). Hubo un caso en el que el FilmArray no identificó el microorganismo causante de la bacteriemia (Acinetobacter baumannii) (2\%) ocasionando terapias antibióticas inapropiadas prolongadas. 
Tabla 2. Comparación de resultados de hemocultivos con resultados del panel BCID FilmArray para la identificación de microorganismos causantes de bacteriemias de cultivos positivos poli y monomicrobianos

\begin{tabular}{|c|c|c|c|c|}
\hline \multirow[t]{2}{*}{ Grupo } & \multicolumn{2}{|l|}{ Resultados hemocultivos preintervención } & \multicolumn{2}{|l|}{ Resultados FilmArray } \\
\hline & & $\mathrm{n}$ & & $\mathrm{n}$ \\
\hline \multirow[t]{5}{*}{ Gram positivos } & SAMR & 6 & SAMR & 6 \\
\hline & SAMS & 7 & SAMS & 7 \\
\hline & SCON & 8 & SCON & 7 \\
\hline & Streptococcus pyogenes & 1 & Streptococcus pyogenes & 1 \\
\hline & Streptococcus pneumoniae & 3 & Streptococcus pneumoniae & 3 \\
\hline \multirow[t]{7}{*}{ Gram negativos } & Klebsiella pneumoniae & 6 & Klebsiella pneumoniae & 6 \\
\hline & Acinetobacter baumannii & 2 & Acinetobacter baumannii & 1 \\
\hline & Serratia marcescens & 1 & Serratia marcescens & 1 \\
\hline & Pseudomonas aeruginosa & 2 & Pseudomonas aeruginosa & 2 \\
\hline & Salmonella tiphy & 3 & Enterobacteriaceae; salmonella tiphy & 1 \\
\hline & Enterobacter cloacae & 1 & Enterobacteriaceae: Salmonella spp. & 1 \\
\hline & Escherichia coli & 4 & Escherichia coli & 3 \\
\hline \multirow[t]{10}{*}{ Polimicrobianos } & \multirow{2}{*}{$\begin{array}{l}\text { Klebsiella pneumoniae y Acinetobacter } \\
\text { baumannii }\end{array}$} & 1 & \multirow[t]{2}{*}{ Klebsiella pneumoniae y Escherichia coli } & 2 \\
\hline & & 1 & & 2 \\
\hline & \multirow{2}{*}{$\begin{array}{l}\text { Klebsiella pneumoniae y Sthaphylococcus } \\
\text { Cromogenes }\end{array}$} & 1 & \multirow[t]{2}{*}{ Klebsiella pneumoniae y Serratia marcescens } & 1 \\
\hline & & 1 & & 1 \\
\hline & \multirow[t]{2}{*}{ Serratia marcescens y Enterobacter cloacae } & 1 & Serratia marcescens y & 2 \\
\hline & & 1 & SAMS & 2 \\
\hline & \multirow[t]{2}{*}{ Serratia marcensens y Escherichia coli } & 1 & \multirow{2}{*}{$\begin{array}{l}\text { Enterobacteriaceae: Salmonella spp y } \\
\text { SAMS }\end{array}$} & 1 \\
\hline & & 1 & & 1 \\
\hline & \multirow[t]{2}{*}{-} & \multirow[t]{2}{*}{-} & \multirow{2}{*}{$\begin{array}{l}\text { Serratia marcescens y Enterobacter cloacae } \\
\text { complex }\end{array}$} & 1 \\
\hline & & & & 1 \\
\hline Hongos & Candida albicans & 1 & Candida albicans & $1^{\mathrm{a}}$ \\
\hline \multirow{3}{*}{$\begin{array}{l}\text { Organismos no identificados } \\
\text { por el panel }\end{array}$} & Burkholderia cepacea & 1 & Burkholderia cepacea & $1^{\mathrm{a}}$ \\
\hline & - & - & Aerococcus viridans & $1^{\mathrm{a}}$ \\
\hline & - & - & Acinetobacter baumannii & $1^{\mathrm{b}}$ \\
\hline
\end{tabular}

SAMR = Staphylococcus aureus meticilino resistente; SAMS = Staphylococcus aureus meticilino sensible; SCoN = Staphylococcus coagulosa negativo; aMicroorganismos no incluidos en el panel para identificación; 'bMicroorganismos incluidos en el panel pero que no fue identificado.

Tabla 3. Comparación de los resultados clínicos y de laboratorio de niños con bacteriemia

\begin{tabular}{|c|c|c|c|c|}
\hline Grupo & Variable & $\begin{array}{l}\text { Grupo Pre-implementación } \\
\qquad \mathrm{n}=(50)(\mathrm{IC} 95 \%)\end{array}$ & $\begin{array}{l}\text { Grupo Pos-implementación } \\
\qquad \mathrm{n}=(50)(\text { IC 95\%) }\end{array}$ & P valor \\
\hline $\begin{array}{l}\text { Resultado } \\
\text { laboratorio }\end{array}$ & $\begin{array}{l}\text { Tiempo de identificación del microorganismo por } \\
\text { Hemocultivo vs FilmArray }\end{array}$ & $70,5(65,2-78,6)$ & $23,0 \quad(12,4-26,7)$ & $0,00 *$ \\
\hline \multirow{4}{*}{$\begin{array}{l}\text { Resultado } \\
\text { clínico }\end{array}$} & Tiempo de inicio de terapia dirigida & $71,9(63,2-77,8)$ & $27,4 \quad(22,3-32,8)$ & $0,00 *$ \\
\hline & $\begin{array}{l}\text { Tiempo de desescalar o suspender antibióticos al } \\
\text { identificar microorganismo FilmArray vs hemocultivo }\end{array}$ & $22,0(6,74-35,6)$ & $6,38(2,76-9,49)$ & 0,92 \\
\hline & Gram positivo & $13,1(3,2-27,4)$ & $2,06(0,11-3,68)$ & 0,45 \\
\hline & Gram negativo & $32,9(5,12-55,4)$ & $11,2 \quad(4,0-16,3)$ & 0,81 \\
\hline
\end{tabular}

*Prueba U de Mann Whitney para variables cuantitativas no normales $(P<0,05)$. 
PREIMPLEMENTACION $70,5 \mathrm{~h}$

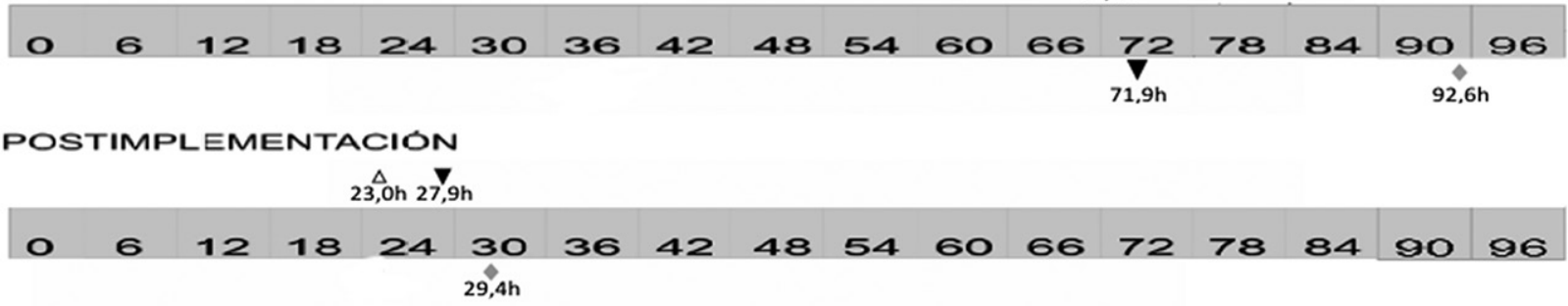

$\Delta$ Ident ficación del Microorganismo Hemocultivo/Film Array $\mathbf{v}$ Inicio de terapia dirigida $\bullet$ Desescalamientc

Figura 1. Comparación de resultados clínicos y de laboratorio de niños con bacteriemia.

Tabla 4. Probabilidad post-prueba, sensibilidad y la especificidad de la tecnología FilmArray para la identificación rápida de bacteriemias

\begin{tabular}{ccccc}
\hline $\begin{array}{c}\text { Probabilidad pre-prueba } \\
\text { estimada }\end{array}$ & Sensibilidad & Especificidad & $\begin{array}{c}\text { Probabilidad post-prueba } \\
\text { positiva \% (IC 95) }\end{array}$ & $\begin{array}{c}\text { Probabilidad post-prueba } \\
\text { negativa \% (IC 95) }\end{array}$ \\
\hline 50,6 & 97,4 & 97,4 & $97,5(88,8-99,5)$ & $2,7(0,6-11,6)$ \\
\hline
\end{tabular}

\section{Discusión}

Este estudio demostró que la rápida identificación de los microorganismos causantes de las bacteriemias utilizando el panel FilmArray BCID incide positivamente en la ejecución del programa URA en la UCIP, debido a que el médico tratante puede orientar el tratamiento antibiótico, suspender las terapias empíricas más rápidamente e iniciar tratamientos adecuados según el microorganismo identificado, aplicando las guías antibióticas institucionales, lo cual a su vez contribuye a disminuir la exposición a antibióticos inadecuados que pueden generar incremento en la resistencia bacteriana y efectos adversos en los pacientes ${ }^{7,8}$.

El tiempo promedio de identificación encontrado en el grupo intervención fue $47 \mathrm{~h}$ menor respecto del grupo control, similar a otros estudios pediátricos en donde el tiempo de identificación con FilmArray fue $42,5 \mathrm{~h}$ menor que la microbiología convencional ${ }^{2}$.

En el grupo intervención la decisión de cambiar antibióticos a terapias dirigidas fue en promedio $44 \mathrm{~h}$ menor y el tiempo de desescalar antibióticos luego de conocer el resultado del FilmArray fue $15 \mathrm{~h}$ menor que el grupo control, decisiones tomadas $24 / 7$ por los médicos tratantes ${ }^{2}$.

Consideramos que los resultados en la disminución de los tiempos para decidir las terapias dirigidas y de desescalar antibióticos, pudieran corresponder a las actividades realizadas como parte del programa URA entre las que se cuentan la sensibilización, capacitación y retroalimentación personalizada del personal médico y de laboratorio clínico.

Es importante resaltar que la tecnología FilmArray por sí sola no garantiza el impacto obtenido en este estudio. Las instituciones que disponen de la misma, deben asegurar que esta se incluya como una herramienta del Programa URA para que los resultados obtenidos se comuniquen de manera oportuna al médico y se realicen los cambios antibióticos pertinentes. En el caso de esta Institución, se acordó con el laboratorio clínico catalogar los resultados del FilmArray como críticos, lo que implica comunicación inmediata y directa con el médico para la toma de decisiones clínicas ${ }^{2,5,6,8,9}$.

La desescalación o suspensión de antibióticos luego de interpretar el FilmArray ocurrió en todos los pacientes, a diferencia del grupo control, siendo similar al estudio de Ray et al. ${ }^{4}$, permitiendo un mejor uso de los antibióticos. Tras la intervención, se reportan terapias dirigidas rápidas, sin esperar el resultado del antibiograma, por lo que las pruebas rápidas de diagnóstico, ayudaron a tomar las decisiones basada en la bacteria identificada ${ }^{8,10,11}$. Además se reporta una menor utilización de antibióticos empíricos inapropiados ${ }^{3,12-14}$, especialmente en el grupo de bacterias Gram positivas ${ }^{15}$, consideramos que lo anterior puede corresponder a 
que los genes de resistencia para bacterias gram positivas testeados por la prueba, como el mecA y van A/B, ayudan al médico a la toma de decisiones para desescalar o suspender antibióticos, lo que no sucede con bacterias gram negativas, puesto que en hospitales con índices de resistencia bajos para blaKPC, la información suministrada sobre la resistencia de carbapenemasas es de menor utilidad que la tipificación de beta lactamasas de espectro extendido (ESBLs/BLEEs). Por lo tanto, la decisión de desescalar carbapenémicos queda sometida al análisis de factores de riesgo de pacientes que puedan tener este tipo de resistencia ${ }^{16}$, especialmente para bacterias como Klebsiella spp., Pseudomonas spp. y Escherichia coli, disminuyendo las posibilidades de desescalar o suspender antibióticos en infecciones causadas por este tipo de bacterias, debido a que el riesgo de terapias antibióticas inapropiadas incrementa los resultados clínicos negativos en este grupo de pacientes $^{13,17}$

El FilmArray presentó en este estudio alta sensibilidad y especificidad para los 24 patógenos, incluyendo cultivos polimicrobianos con adecuada concordancia, al compararlo con la microbiología convencional ${ }^{2,7,18-22}$. Southern et al. ${ }^{18}$, no identificaron 8 de los microorganismos presentes en el panel, reafirmando la adecuada sensibilidad y especificidad para los microorganismos incluidos en el FilmArray BCID. Los pacientes que presentaron bacteriemias por Salmonella spp., y Salmonella typhi fueron identificados por el panel como enterobacteriaceae y presentaron retrasos en el inicio de la terapia dirigida, evidenciando nuevamente la importancia de la identificación rápida de los microorganismos causantes de bacteriemias para una adecuada elección y duración del tratamiento antibiótico. La sensibilidad y especificidad en nuestro estudio para los genes de resistencia fue de $100 \%$ con relación a microbiología convencional; estos resultados se asemejan al estudio multicéntrico de Salimnia et al. ${ }^{23,24}$ quienes encontraron una sensibilidad de 98,4 y especificidad 98,3 en mecA y del $100 \%$ para van A/B y blaKPC ${ }^{25,26}$, lo cual genera confianza en la interpretación del panel FilmArray BCID.

Nuestro estudio tiene algunas limitaciones, empezando por las propias de un estudio retrospectivo. Las variables sociodemográficas no están controladas ya que se tuvieron en cuenta los pares de la misma especie del germen identificado. Como en el periodo pre-in- tervención no se ejecutó el programa URA no se pueden generalizar los resultados obtenidos en el periodo post-intervención para los tiempos de terapias dirigidas y de desescalar o suspender antibióticos. Al ser realizado en un centro único, la muestra fue relativamente pequeña, por lo que algunas de las variables muestran tendencias sin llegar a la significancia estadística y se debe incluir más pacientes para confirmarlas.

Pese a ello, queremos destacar que éste es uno de los pocos estudios realizados en población pediátrica en Latinoamérica, que evalúa la efectividad del FilmArray para la identificación de bacteriemias, y que muestra las ventajas de disponer de esta prueba en conjunto con actividades de uso racional de antibióticos.

En conclusión, la tecnología FilmArray es una herramienta útil, que contribuye en los programas URA a la toma de decisiones en pacientes críticos con bacteriemia, y que nos permite identificar rápidamente los microorganismos causantes con una elevada sensibilidad y especificidad, generando confianza en la toma de decisiones referentes a ajustes del tratamiento antibiótico, permitiendo al médico formular terapias antibióticas dirigidas y disminuyendo la exposición de los pacientes a antibióticos empíricos inapropiados.

\section{Responsabilidades Éticas}

Protección de personas y animales: Los autores declaran que los procedimientos seguidos se conformaron a las normas éticas del comité de experimentación humana responsable y de acuerdo con la Asociación Médica Mundial y la Declaración de Helsinki.

Confidencialidad de los datos: Los autores declaran que han seguido los protocolos de su centro de trabajo sobre la publicación de datos de pacientes.

Derecho a la privacidad y consentimiento informado: Los autores han obtenido el consentimiento informado de los pacientes y/o sujetos referidos en el artículo. Este documento obra en poder del autor de correspondencia.

\section{Conflicto de intereses}

Los autores declaran no tener conflicto de intereses. 


\section{Referencias}

1. Garnacho J, Gutiérrez A, Escoresca A. et al. De-escalation of empirical therapy is associated with lower mortality in patients with severe sepsis and septic shock. Intensive Care Med. 2014;40:32-40.

2. Messacar K, Hurst A, Child J, et al. Clinical impact and provider acceptability of real-time antimicrobial stewardship decision support for rapid diagnostics in children with positive blood culture. J of the Pediatr Infect Dis Soc. 2017;6(3):26774.

3. Pardo J, Klinker K, Borgert S, Butler B, Giglio P, Rand K. Clinical and economic impact of antimicrobial stewardship interventions with the FilmArray blood culture identification panel. Diagn Microbiol and Infect Dis. 2016;84:159-64.

4. Ray S, Phil M, Drew R, Hardiman F, Pizer B, Riordan A. Rapid identification of microorganisms by FilmArray blood culture identification panel improves Clinical management in children. Pediatr Infect Dis J. 2016;35:e134-e138.

5. Doern $\mathrm{CH}$. The confounding role of antimicrobial stewardship programs in understanding the impact of technology on patient care. J Clin Microbiol. 2016;54(10):2420-3.

6. Maurer Fl, Christner M, Hentschke M, Rohde H. Advances in rapid identification and susceptibility testing of bacteria in the clinical microbiology laboratory: implications for patient care and antimicrobial stewardship programs. Infect Dis Rep. 2017;9:18-27.

7. Blaschke A, Heyrend C, Byington C, et al. Rapid identification of pathogens from positive blood cultures by multiplex polymerase chain reaction using the FilmArray system. Diagn Microbiol and Infect Dis. 2012;74:349-55.

8. MacVane S, Nolte F. Benefits of adding a rapid PCR-Based blood culture identification panel to an established antimicrobial stewardship program. J of Clin Microbiol. 2016;54(10):2455-63.

9. Reuter CH, Palac HL, Kociolek LK, et al. Ideal and actual impact of rapid diagnostic testing and antibiotic stewardship on antibiotic prescribing and clinical outcomes in children with positive blood cultures. Pediatr Infect Dis J. 2019;38(2):131-7.

10. Messacar K, Parker S, Todd J, Domínguez S. Implementation of rapid molecular infectious disease diagnostics: the role of diagnostic and antimicrobial stewardship. J of Clin Microbiol. 2017;55(3):715-23.

11. Markley D, Bernard Sh, Bearman G, Stevens M. De-escalating antibiotic use in the inpatient setting: strategies, controversies, and challenges. Curr Infect Dis Rep. 2017;19(4):1-17.

12. Sullivan K. Rapid molecular panels: what is the best interest of the patient? A review of patient outcome studies for multiplex panels used in bloodstream, respiratory and neurological infections. Clin Microbiol newslett. 2017;39(16):125-9.

13. Raman G, Avendano E, Berger S, Menon V. Appropriate initial antibiotic therapy in hospitalized patients with gramnegative infections: systematic review and meta-analysis. BMC infect Dis. 2015; 15:395.

14. Banerjee R, Teng Chr, Cunningham S, et al. Randomized trial of rapid multiplex polymerase chain reaction-based blood culture identification and susceptibility testing. Clin Infect Dis. 2015;61(7):107180 .

15. Tseng A, Kasule S, Rice F, Mi L, Chan L, Seville M, et al. Is it actionable? An evaluation of the rapid PCR-based blood culture identification panel on the management of Gram-positive and gram-negative blood stream infections. Open Forum Infect Dis. 2018;5: https:// www.ncbi.nlm.nih.gov/pmc/articles/ PMC6288766/última visita 05-112018.

16. Bookstaver PB, Nimmich EB, Smith TJ, et al. Cumulative effect of an antimicrobial stewardship and rapid diagnostic testing bundle on early streamlining of antimicrobial therapy in Gram-negative bloodstream infections. Antimicrob agents chemother. 2017;61(9):1-10.

17. Riedel S, Carrol K. Early identification and treatment of pathogens in sepsis molecular diagnostics and antibiotic choice. Clin Chest Med. 2016;37: 191-207.

18. Southern T, VanSchooneveld T, Bannister $\mathrm{D}$, et al. Implementation and performance of the Biofire FilmArray blood culture identification panel with antimicrobial treatment recommendations for bloodstream infections at a Midwestern academic tertiary hospital. Diagn Microbiol and Infect Dis. 2015;81:96-101.

19. Altun $\mathrm{O}$, Almuhayawi M, Ullberg $\mathrm{M}$, O"zensi V. Clinical evaluation of the FilmArray blood culture identification panel in identification of bacteria and yeasts from positive blood culture bottles. J of Clin Microbiol. 2013;51(12):4130-6.

20. Fiori B, D'inzeo T, Giaquinto A, et al. Optimized use of the maldi biotype system and the FilmArray BCDI panel for direct identification of microbial pathogens from positive blood cultures. J Clin Microbiol. 2016;54(3):576-84.

21. Minejima E, Wong-Beringer A. Implementation of rapid diagnostics with antimicrobial stewardship. Exp Rev of anti-infect there. (internet) september 2016; http://dx.doi.org/10.1080/14787210. 2016.1233814.

22. Salimnia H, Fairfax M, Lephart P, et al. Evaluation of the FilmArray Blood Culture Identification Panel: Results of a Multicenter Controlled Trial. J of Clin Microbiol: 2016;54(3):687-98.

23. Timbrook T, Spivak E, Hanson K. Current and future opportunities for rapid diagnostics in antimicrobial stewardship. Med. The clinics. com. 2018;102:899-911. https://doi. org/10.1016/j.mcna.2018.05.004, última visita 09-02-2019.

24. Zheng X, Polanco W, Carter D, Shulman S. Rapid identification of pathogens from pediatric blood cultures by use of the FilmArray blood culture identification panel. J of Clin Microbiol: 2014;52(12):4368-71.

25. Pilakos E, Andratos N, Shehaded P, Ziakas P, Milonakis E. The costeffectiveness of rapid diagnostic testing for the diagnosis of bloodstream infections with or without antimicrobial stewardship. Clin Microbiol Rev. 2018;31(3):1-22.

26. Fimbres A, Olson J, Hersh A, et al. A retrospective study of the impact of rapid diagnostic testing on time to pathogen identification and antibiotic use for children with positive blood cultures. Infect Dis Ther. 2016;5:555-70. 